\title{
Performance Analysis of Energy Harvesting UAV Selection
}

\author{
Khuong Ho-Van $\mathbb{D}^{1,2}$ and Thiem Do-Dac $\mathbb{D}^{1,2,3}$ \\ ${ }^{1}$ Ho Chi Minh City University of Technology (HCMUT), 268 Ly Thuong Kiet Street, District 10, Ho Chi Minh City, Vietnam \\ ${ }^{2}$ Vietnam National University Ho Chi Minh City, Linh Trung Ward, Thu Duc District, Ho Chi Minh City, Vietnam \\ ${ }^{3}$ Thu Dau Mot University, 6 Tran Van On Street, Phu Hoa Ward, Thu Dau Mot City, Binh Duong Province, Vietnam
}

Correspondence should be addressed to Thiem Do-Dac; thiemdd@tdmu.edu.vn

Received 6 January 2021; Revised 20 February 2021; Accepted 26 February 2021; Published 27 March 2021

Academic Editor: Tran Manh Hoang

Copyright (c) 2021 Khuong Ho-Van and Thiem Do-Dac. This is an open access article distributed under the Creative Commons Attribution License, which permits unrestricted use, distribution, and reproduction in any medium, provided the original work is properly cited.

\begin{abstract}
In an UAV- (unmanned aerial vehicle-) aided relaying system, the transmitted signal is exposed to free space in two transmission hops which may be overheard by eavesdroppers. Accordingly, physical layer security should be exploited to improve information security. This paper analyzes both (security and reliability) performance aspects of such a system where only one UAV among multiple UAVs, all capable of harvesting energy from radio frequency signals, is adopted. Towards this end, the tight approximated and exact closed-form expressions of the outage probability at the legitimate destination and the intercept probability at the eavesdropper are first derived. Then, Monte-Carlo simulations are conducted to verify the derived expressions. Based on these expressions, the protected zone of the selected UAV is also proposed through an exhaustive search. Finally, various results are provided to illustrate the impact of key operation parameters on the system performance and the efficacy of the UAV selection.
\end{abstract}

\section{Introduction}

Unmanned aerial vehicles (UAVs) have advantages of high mobility, low cost, and on-demand deployment and, thus, have found multiple important applications in recent years, especially in the telecommunications sector [1]. UAVs, which can transport and operate as mobile mini base stations in the air, are utilized for providing wireless services [2]. Unlike traditional relays that are fixed at specific locations, UAVs can change their positions in order to provide a large coverage region [3]. Moreover, using UAVs has recently emerged as an idea to respond to high localized traffic demands in next generation cellular networks [4].

Despite its promising benefits, UAV-enabled communications face many challenges. The big challenge is the broadcast nature of wireless communications, causing air-to-ground communications to expose to free space. Therefore, eavesdroppers can overhear the transmitted signals which are inside the coverage region of the aerial station, inducing unsecured transmission. To secure wireless communications, physical layer security has recently been exploited as an alternative- and-complementary solution [5]. Indeed, given advances in computation power and resources, eavesdroppers are able to regenerate secret keys to decode encrypted information, and, hence, security in multiple layers including the physical layer should be applied to better protect legitimate information. In addition, different from terrestrial channels where the locations of the base stations (BSs) are fixed on the twodimensional (2D) ground plane, air-to-ground channels vary significantly with UAVs' mobility. This causes difficulties in channel modelling (for example, the position of the UAV is represented by the three-dimensional (3D) Cartesian coordinate instead of the $2 \mathrm{D}$ coordinate for terrestrial $\mathrm{BSs}$ ), performance analysis, system design optimization, etc. However, additional free dimensions of the UAV's coordinate offer key opportunities in UAV-aided communications in finding optimum locations to deploy aerial stations which achieve reliable-and-secured channels. Moreover, the energy of UAVs available for powering onboard electronics is limited because of using batteries as a source of power. The solution to the energy limitation of UAVs is to equip UAVs with energy harvesters, which can scavenge energy in radio frequency (RF) 
signals to self-power their operations, eventually keeping reliable connection of UAV communications [6]. Furthermore, in the case of multiple UAVs, how to select an UAV as a relay for reliable communications to the legitimate receiver and secured transmission against eavesdroppers is another challenge. It is recalled that the UAV selection can reduce the burden of energy consumption and signal synchronization and considerably save transmission bandwidth as compared to using all UAVs as relays [7].

1.1. Related Works. Some initial works on secrecy performance analysis of UAV-based communications systems are listed as [8-14]. More specifically, [8] analyzed the secrecy outage probability (SOP) of a standard UAV-based communications system where a UAV plays a role as a legitimate aerial transmitter who communicates with the legitimate on-ground receiver in the presence of multiple eavesdroppers who are randomly located. In $[9,10]$, the system model in [8] was reconsidered without any performance analysis. Instead, [9] optimized the average secrecy rates of the UAV-toground and ground-to-UAV transmissions by jointly optimizing the transmit power of the legitimate user and the trajectory of the UAV, while [10] optimized the position of the UAV by maximizing the probability of nonzero secrecy capacity. Different from [8-10], the authors in [11] studied all aerial users. Moreover, [11] proposed an analytical framework on the average secrecy capacity and SOP, which takes into account random distribution of the wire-tapping UAVs and the legitimate UAV receiver. In [12], Han-Kobayashi signaling was applied to secure the standard UAV-based multiuser communications system where an UAV plays a role as a legitimate aerial transmitter who communicates with multiple legitimate on-ground receivers in the presence of an eavesdropper, and the users' worst secrecy throughput was maximized by jointly optimizing bandwidth, the time-fraction, and power allocation. In [13], a swarm of UAVs cooperates with each other to eavesdrop information communicated between the on-ground legitimate sourcedestination pair, and eavesdropped information at different UAVs is combined using the maximum ratio combining (MRC) or the selection combining (SC) for better eavesdropping performance. The secrecy capacity was analyzed in an approximated form in [13]. In [14], a swarm of UAVs can be divided into two types: relay and jammer. As a relay, the selected UAV receives signals from the on-ground source station and forwards the processed signals to the on-ground destination station, while as jammers, UAVs transmit jamming signals to interfere the eavesdroppers. The selected UAV is the one that correctly decodes the source signal and has the lowest path loss to the destination. The SOP was analytically approximated in [14] without considering the energy harvesting at UAVs.

Some works also focused on constructing a swarm of UAVs to increase the probability of the end-to-end (E2E) connection [15-17]. In [15], a swarm of slave UAVs transmits their information which is relayed by a master UAV to an on-ground receiver. The outage probability was analyzed in [15] for Nakagami- $m$ channels. In [16], multiple UAVs are exploited as (amplify-and-forward (AF) or decode-and- forward (DF)) relay in two (single multihop link or multiple dual-hop links) network topologies. The performance of the UAV-aided relaying system in [16] was also analyzed in terms of bit error rate and outage probability. Additionally, UAVs are optimally positioned with the maximum E2E signal-to-noise ratio (SNR). In [17], the distances from a swarm of UAVs as AF relays to the multiantenna source station and the destination station are optimized for the maximum channel capacity. It should be noted that both [16, 17] did not pay attention to the security problem in UAVaided relaying systems when eavesdroppers are present.

1.2. Contributions. In contrast to existing literature, this paper analyzes both the secrecy performance in terms of the intercept probability (IP) and the connection reliability in terms of the outage probability (OP). A swarm of UAVs is also considered in this paper. However, different from [13-17], this paper selects only one UAV for relaying the legitimate information from the on-ground source station to the on-ground destination station, and all UAVs under consideration are capable of harvesting energy from the source station's signals. The energy harvesting (EH) capable UAV selection can mitigate the requirements of signal synchronization, transmission bandwidth, and power limitation of UAVs.

Here are the key contributions of our study:

(i) Propose an UAV selection scheme to adopt only one UAV as a relay from a swarm of UAVs, all capable of harvesting energy from RF signals, for reliableand-secured communications of the legitimate source-destination pair under the eavesdropping of the wire-tapper without a heavy burden of transmission bandwidth, power consumption, and signal synchronization

(ii) Suggest the tight approximated and exact closedform expressions (in the sequel, two terms ("approximated closed-form expression" and "exact closedform expression") are used frequently. The former refers to the expression which is represented in the explicit form (i.e., no integral is left) and derived from some approximated quantities. Meanwhile, the latter refers to the expression which is represented in the explicit form and derived from all exact quantities) of OP and IP for the EH UAV selection under consideration of different availability degrees of wire-tapped information, path-loss, and Rayleigh fading for air-to-ground communication links

(iii) Find the protected zone of the selected UAV not only to provide reliable communications of the legitimate source-destination pair but also to secure these communications against the eavesdropper

(iv) Provide various results to have insights into the impact of the main parameters of transmit power, time splitting factor, and the position of the selected UAV on the connection reliability and the secrecy performance of UAV-aided relaying systems 
1.3. Paper Structure. The paper continues as follows. Section 2 presents the system model under investigation. Then, IP and $\mathrm{OP}$ are analyzed in detail in Section 3. Next, the protected zone of the selected UAV as a relay is outlined in Section 4. Subsequently, the Monte-Carlo simulation is conducted to verify the theoretical analysis in Section 5. This section also provides various results on the performance of the EH UAV selection with respect to key operation parameters. Finally, Section 6 summarizes and concludes our work.

\section{System Model}

Consider an UAV-aided relaying system as depicted in Figure 1(a), where an on-ground source station $S$ communicates with an on-ground destination station $D$. Assuming that direct communication between $S$ and $D$ is blocked due to some reasons such as long distance, deep fading, and strong shadowing. Therefore, this communication needs the aid of the best UAV, namely, $R_{b}$, which is selected from $M$ available UAVs operating as energy harvesting AF relays. Let the $i^{\text {th }}$ UAV be $R_{i}$, where $i \in \Phi_{R}=\{1,2, \cdots, M\}$. Then, the best UAV is defined as the one whose SNR from the source signal is larger than that of any other $\operatorname{UAV} R_{j}$ with $j \in \Phi_{R} \backslash b$ for the most successfully decoding of the source signal. An eavesdropper $E$ tries to wire-tap information transmitted by $S$ and $R_{b}$.

2.1. Channels. Assuming that all the channels are independent, flat, and Rayleigh-distributed and remain unchanged during each transmission but independently vary from one transmission to another. The channel coefficient of the $u-$ $v$ communication link is denoted as $h_{u v}$, where $u \in\left\{S, R_{i}\right\}$ and $v \in\left\{R_{i}, D, E\right\}$. Then, the probability density function (PDF) and the cumulative distribution function (CDF) of the channel gain of the $u-v$ communication link are correspondingly expressed as

$$
\begin{gathered}
f_{\left|h_{u v}\right|^{2}}(x)=\frac{1}{\bar{\gamma}_{u v}} e^{-x / \bar{\gamma}_{u v}}, \\
F_{\left|h_{u v}\right|^{2}}(x)=1-e^{-x / \bar{\gamma}_{u v}},
\end{gathered}
$$

where $x \geq 0$ and $\bar{\gamma}_{u v}=\Xi\left\{\left|h_{u v}\right|^{2}\right\}$ are the mean of the random variable $\left|h_{u v}\right|^{2}$, also known as the fading power of the $u-v$ link; $\Xi$ is the expectation operator.

Accounting for the path loss [16], the fading power of the $u-v$ communication link can be modelled as $\bar{\gamma}_{u v}=\kappa d_{u v}^{-\tau}$, where $\kappa=\left(4 \pi f_{c} / c\right)^{-2}, \tau$ is the path loss exponent, $d_{u-v}$ is the distance of the $u-v$ communication link, $f_{c}$ is the carrier frequency $[\mathrm{Hz}]$, and $c=3 \times 10^{8}[\mathrm{~m} / \mathrm{s}]$ is the speed of light.

2.2. Signals. Given the fact that the energy of UAVs available for powering onboard electronics is limited because of using batteries as a source of power, UAVs should be self-powered by scavenging energy from RF signals of the source station and use harvested energy to amplify-and-forward their received signals to the destination station. As seen in Figure 1(b), the total time $T$ for the end-to-end transmission of a message is divided into three phases. During the first phase of $\alpha T$ with $0<\alpha<1$ being the time splitting factor, UAVs scavenge energy from RF signals of the source station. The energy which the UAV $R_{i}, i \in \Phi_{R}$, harvests in this phase is $E H_{R_{i}}=\eta \alpha T P_{S}\left|h_{S R_{i}}\right|^{2}$, where $P_{S}$ denotes the transmit power of the source station, $\eta$ is the energy conversion efficiency, and $h_{\mathrm{SR}_{i}}$ is the channel coefficient of the $S-R_{i}$ communication link. In the second phase of $(1-\alpha) T / 2$, the source station transmits its signal to UAVs. At the end of this phase, the best UAV $R_{b}$ is selected according to the criterion as mentioned at the beginning of this section. The best UAV operates as an AF relay which amplifies the source signal and transmits the amplified signal to the destination station in the third phase of $(1-\alpha) T / 2$.

In the second phase, the received signal at the selected UAV $R_{b}$ can be expressed as $y_{R_{b}}=x_{S} h_{\mathrm{SR}_{b}}+n_{R_{b}}$, where $x_{S}$ is the signal transmitted by $S$ with the transmit power $P_{S}=\Xi$ $\left\{\left|x_{S}\right|^{2}\right\}, h_{\mathrm{SR}_{b}}$ is the channel coefficient of the $S-R_{b}$ communication link, and $n_{R_{b}}$ denotes the noise at $R_{b}$. Without loss of generality, the noise at the receiver $v$, where $v \in\left\{R_{i}, D, E\right\}$ and $i \in \Phi_{R}$, is assumed to be additive white Gaussian noises (AWGN) with zero mean and variance $N_{0}$, i.e., $\Xi\left\{n_{v}\right\}=0$ and $\Xi\left\{\left|n_{v}\right|^{2}\right\}=N_{0}$. Then, the instantaneous SNR of the $S$ $-R_{b}$ link is

$$
\gamma_{\mathrm{SR}_{b}}=\psi\left|h_{\mathrm{SR}_{b}}\right|^{2}
$$

where $\psi=P_{S} / N_{0}$ stands for the normalized SNR.

In the third phase, the best UAV $R_{b}$ consumes its harvested energy to forward the amplified source signal $x_{R_{b}}$ to the destination station with the following transmit power:

$$
P_{R_{b}}=\frac{E H_{R_{b}}}{(1-\alpha) T / 2}=\xi \eta P_{S}\left|h_{\mathrm{SR}_{b}}\right|^{2},
$$

where $\xi=2 \alpha /(1-\alpha)$ is defined as the time-switching coefficient.

The amplified source signal $x_{R_{b}}$, which is transmitted from $R_{b}$ to $D$, is expressed as $x_{R_{b}}=\beta y_{R_{b}}$, where the amplification coefficient $\beta$ is computed such that the transmit power of $R_{b}$ is $P_{R_{b}}$ in (4), i.e.,

$$
\beta=\sqrt{\frac{\Xi\left\{\left|x_{R_{b}}\right|^{2}\right\}}{\Xi\left\{\left|y_{R_{b}}\right|^{2}\right\}}}=\sqrt{\frac{P_{R_{b}}}{P_{S}\left|h_{\mathrm{SR}_{b}}\right|^{2}+N_{0}}} .
$$

Therefore, the received signal at the destination station is expressed as $y_{D}=x_{R_{b}} h_{R_{b} D}+n_{D}=\beta x_{S} h_{\mathrm{SR}_{b}} h_{R_{b} D}+\beta h_{R_{b} D} n_{R_{b}}+$ $n_{D}$, where $h_{R_{b} D}$ is the channel coefficient of the $R_{b}-D$ communication link, and $n_{D}$ denotes the noise at $D$. Then, the end-to-end SNR of the $S-R_{b}-D$ communication link can be expressed as 


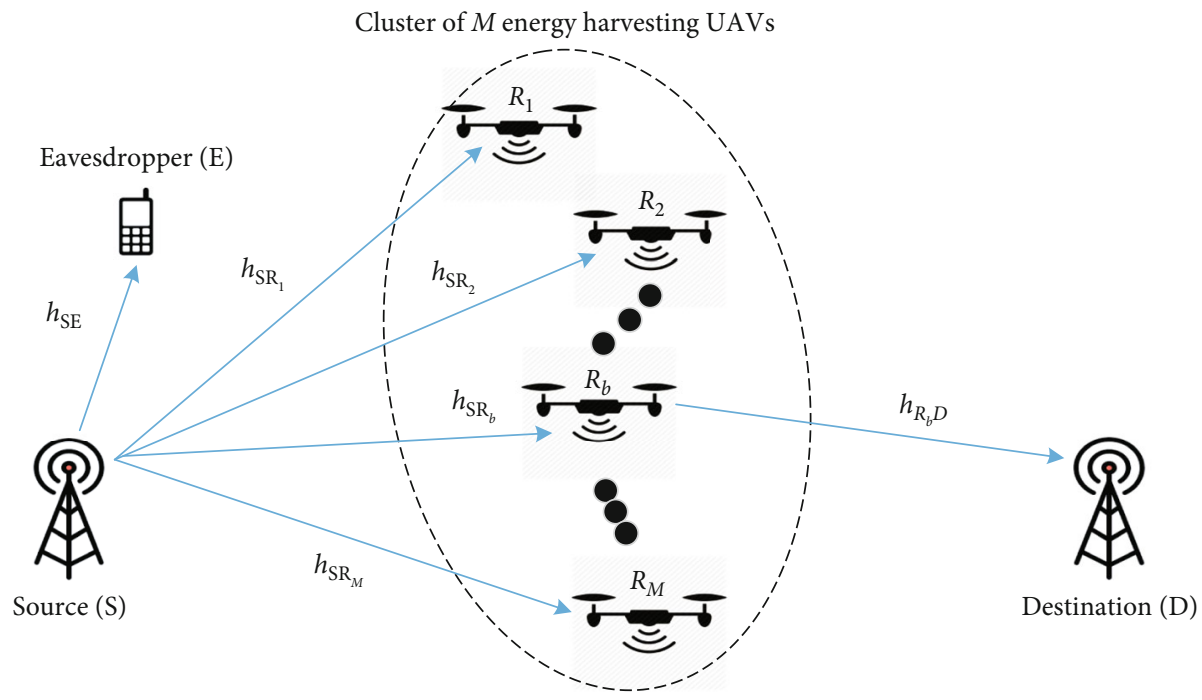

(a)

\begin{tabular}{|c|c|c|}
\hline Energy harvesting & Source to UAVs & Selected UAV to destination \\
\hline
\end{tabular}

(b)

FIGURE 1: UAV-aided relaying system in the presence of eavesdropper.

$$
\gamma_{D}=\frac{\beta^{2} P_{S}\left|h_{\mathrm{SR}_{b}}\right|^{2}\left|h_{R_{b} D}\right|^{2}}{\beta^{2}\left|h_{R_{b} D}\right|^{2} N_{0}+N_{0}}
$$

Substituting (5) into (6) and after some simplifications, one obtains

$$
\gamma_{D}=\frac{P_{S}\left|h_{\mathrm{SR}_{b}}\right|^{2}\left|h_{R_{b} D}\right|^{2}}{\left|h_{R_{b} D}\right|^{2} N_{0}+\left(N_{0} P_{S}\left|h_{\mathrm{SR}_{b}}\right|^{2} / P_{R_{b}}\right)+\left(N_{0}^{2} / P_{R_{b}}\right)} .
$$

Since the transmit power $P_{S}$ is much greater than the noise power $N_{0}, N_{0}^{2} / P_{R_{b}} \simeq 0$. As such, (7) can be approximated tightly as

$$
\gamma_{D} \simeq \frac{P_{S}\left|h_{\mathrm{SR}_{b}}\right|^{2}\left|h_{R_{b} D}\right|^{2}}{\left|h_{R_{b} D}\right|^{2} N_{0}+\left(N_{0} P_{S}\left|h_{\mathrm{SR}_{b}}\right|^{2} / P_{R_{b}}\right)} .
$$

Substituting $P_{R_{b}}$ in (4) into (8), one obtains

$$
\gamma_{D} \simeq \frac{\xi \eta \psi\left|h_{\mathrm{SR}_{b}}\right|^{2}\left|h_{R_{b} D}\right|^{2}}{\xi \eta\left|h_{R_{b} D}\right|^{2}+1}
$$

2.3. Availability Degree of Eavesdropped Information. The eavesdropper $E$ can receive signals in either one phase (second phase) or two phases (second and third phases). Depending on the availability degree of eavesdropped information, the corresponding scenarios are considered.
2.3.1. Scenario 1: E Receives Signals Only from $S$ in the Second Phase. Due to some reason, $E$ may not receive signals from the best UAV $R_{b}$. This is Scenario 1. In this scenario, the received signal at $E$ from $S$ is given by $y_{S E}=x_{S} h_{S E}+n_{E}$, where $h_{S E}$ is the channel coefficient of the $S-E$ communication link, and $n_{E}$ denotes the noise at $E$. Then, the SNR at $E$ is the SNR of the $S-E$ link as

$$
\gamma_{E o}=\gamma_{\mathrm{SE}}=\psi\left|h_{\mathrm{SE}}\right|^{2}
$$

which is similar to (3).

2.3.2. Scenario 2: E Receives Signals from $S$ in the Second Phase and $R_{b}$ in the Third Phase. This scenario allows $E$ to combine both signals from $S$ and $R_{b}$ for higher intercept probability. It is recalled that the received signal at $E$ over the $R_{b}-E$ communication link is given by $y_{R_{b} E}=x_{R_{b}} h_{R_{b} E}+n_{E}$, which results in the SNR of $\gamma_{R_{b} E}=P_{R_{b}}\left|h_{R_{b} E}\right|^{2} / N_{0}$. Therefore, two signals $\left(y_{\mathrm{SE}}\right.$ and $y_{R_{b} E}$ ) are available at $E$ in Scenario 2. Based on these two signals, $E$ can combine them by two methods depending on implementation complexity. Given affordable implementation complexity, E can perform selection combining (SC) in [18] to select the received signal with the higher SNR. Therefore, the SC method offers the instantaneous SNR at $E$ as

$$
\gamma_{\mathrm{Esc}}=\max \left(\gamma_{\mathrm{SE}}, \gamma_{R_{b} E}\right)
$$

For higher implementation complexity (it is obvious that the MRC technique is more complicated but better than the $\mathrm{SC}$ one), $E$ can perform maximum ratio combining (MRC) 
in [18] to sum the SNRs of both $\left(S-E\right.$ and $\left.R_{b}-E\right)$ communication links, resulting in the instantaneous SNR at $E$ as

$$
\gamma_{E m r c}=\gamma_{\mathrm{SE}}+\gamma_{R_{b} E}
$$

Just remind that the SNR of $R_{b}-E$ link is of the same form as the SNR of $R_{b}-D$ link in (9). Therefore, $\gamma_{R_{b} E}$ is approximated tightly as

$$
\gamma_{R_{b} E} \simeq \frac{\xi \eta \psi\left|h_{\mathrm{SR}_{b}}\right|^{2}\left|h_{R_{b} E}\right|^{2}}{\xi \eta\left|h_{R_{b} E}\right|^{2}+1} .
$$

\section{Performance Analysis}

To numerically evaluate the performance of the energy harvesting UAV selection, the tight approximated and exact closed-form expressions of the outage probability and the intercept probability are derived in the following.

It is recalled that among $M$ UAVs, only the best UAV $R_{b}$ is selected according to the partial selection principle which offers the highest SNR over all the source-UAV communication links. Therefore, the index of the best UAV can be denoted as $b=\arg \max _{i \in \Phi_{R}} \gamma_{S R_{i}}$. Then, the SNR at $R_{b}$ is equivalently represented as $\gamma_{\mathrm{SR}_{b}}=\max _{i \in \Phi_{R}} \gamma_{S R_{i}}$. For ease of presentation (the case that all the UAVs are heterogeneously located is straightforwardly extended. Indeed, Appendix D outlines how to derive the tight approximated closed-form expressions of the outage probability and the intercept probability for this case. However, it is noted that the final expressions will be untidy, and, hence, they are not really convenient for computer programming and result illustrations. Accordingly, this paper presents only the case of homogeneous locations of UAVs. Interested readers can follow Appendix D to obtain the analysis for the case of heterogeneous locations of UAVs, for their own purposes), all the UAVs are assumed to be homogeneously located, resulting in $\bar{\gamma}_{\mathrm{SR}_{1}}=\bar{\gamma}_{\mathrm{SR}_{2}}=\cdots=$ $\bar{\gamma}_{\mathrm{SR}_{M}}=\bar{\gamma}_{\mathrm{SR}}$ which is the fading power of the S-UAV communication link. By denoting $X=\max _{i \in \Phi_{R}}\left|h_{\mathrm{SR}_{i}}\right|^{2}$, one obtains its statistics in the following lemma.

Lemma 1. The CDF and PDF of $X$ are correspondingly given by

$$
\begin{gathered}
F_{X}(x)=\sum_{q=0}^{M}(-1)^{q} C_{M}^{q} e^{-q / \bar{\gamma}_{S R} x}, \\
f_{X}(x)=\frac{M}{\bar{\gamma}_{S R}} \sum_{q=0}^{M-1}(-1)^{q} C_{M-1}^{q} e^{-(q+1) / \bar{\gamma}_{S R} x}
\end{gathered}
$$

where $x \geq 0$ and $C_{M}^{q} \triangleq M ! / q ! /(M-q) !$ is the binomial coefficients.

Proof. Please see Appendix A.
Based on information theory, the end-to-end SNR of the $S-R_{b}-D$ communication link, $\gamma_{D}$, should be greater than a threshold $\gamma_{\text {th }}$ to keep a reliable end-to-end connection (the channel capacity that $D$ can achieve is $C_{D}=(1-\alpha) / 2 \log$ $\left(1+\gamma_{D}\right)$. Therefore, $D$ successfully recovers the source information if its channel capacity is greater than the required spectral efficiency $C_{\text {th }}$. This is equivalent to $\gamma_{D}$ greater than $\left.\gamma_{\text {th }}=2^{\left(2 C_{\text {th }} /(1-\alpha)\right)}-1\right)$. The probability that $\gamma_{D}$ is not greater than that threshold is known as the outage probability, i.e., $O P=\operatorname{Pr}\left\{\gamma_{D}<\gamma_{\text {th }}\right\}=F_{\gamma_{D}}\left(\gamma_{\text {th }}\right)$. Using $\gamma_{D}$ in (9), one can rewrite $F_{\gamma_{D}}\left(\gamma_{\text {th }}\right)$ in a compact form as

$$
F_{\gamma_{D}}\left(\gamma_{\mathrm{th}}\right) \simeq \operatorname{Pr}\left\{\frac{\xi \eta X Y}{\xi \eta Y+1}<\frac{\gamma_{\mathrm{th}}}{\psi}\right\}
$$

where $X=\left|h_{\mathrm{SR}_{b}}\right|^{2}=\max _{i \in \Phi_{R}}\left|h_{\mathrm{SR}_{i}}\right|^{2}$ and $Y=\left|h_{R_{b} D}\right|^{2}$.

Lemma 2. The tight approximated closed-form expression (this expression is approximated because it is derived from the approximated $\gamma_{D}$ in (9). Nonetheless, the approximation in (9) is tight, and, hence, (17) matches simulation results as shown in Section5) of the outage probability of the energy harvesting $U A V$ selection is given by

$$
\begin{gathered}
O P=F_{\gamma_{D}}\left(\gamma_{t h}\right) \simeq 1-\frac{2 M}{\psi \bar{\gamma}_{S R}} \sum_{q=0}^{M-1}(-1)^{q} C_{M-1}^{q} e^{-(q+1) \gamma_{t h} / \psi \bar{\gamma}_{S R}} \\
\sqrt{\frac{\psi \bar{\gamma}_{S R} \gamma_{t h}}{(q+1) \xi \eta \bar{\gamma}_{R D}}} K_{1}\left(2 \sqrt{\frac{(q+1) \gamma_{t h}}{\xi \eta \psi \bar{\gamma}_{S R} \bar{\gamma}_{R D}}}\right)
\end{gathered}
$$

where $\bar{\gamma}_{R_{1} D}=\bar{\gamma}_{R_{2} D}=\cdots=\bar{\gamma}_{R_{M} D}=\bar{\gamma}_{R D}$ and $K_{1}(\cdot)$ is the modified Bessel function of the second kind and the first order.

Proof. Please see AppendixB.

Also based on information theory, the eavesdropper successfully decodes the source information from its received signals (or $E$ intercepts the source information) if its SNR is larger than a threshold (the channel capacity that $E$ can achieve is $C_{E}=(1-\alpha) / 2 \log \left(1+\gamma_{E}\right)$. Therefore, $E$ successfully recovers the source information if its channel capacity is greater than the required spectral efficiency $C_{I}$. This is equivalent to $\gamma_{E}$ greater than $\gamma_{I}=2^{2 C_{I} /(1-\alpha)}-1$. The probability that such an event happens is defined as the intercept probability: $\mathrm{IP}=\operatorname{Pr}\left\{\gamma_{E} \geq \gamma_{I}\right\}=1-\operatorname{Pr}\left\{\gamma_{E}<\gamma_{I}\right\}$. It is noted that $\gamma_{E}$ can take the value in (10) or (11) or (12), depending on the availability degree of the eavesdropped information at $E$.

The following derives the IP expressions for every values that $\gamma_{E}$ can take.

3.1. Scenario 1: E Receives Signals Only from $S$ in the Second Phase. In this scenario, $\gamma_{E}=\gamma_{E o}$ is given in (10). Therefore, the IP is expressed as $\operatorname{IP}_{o}=1-\operatorname{Pr}\left\{\psi\left|h_{\mathrm{SE}}\right|^{2}<\gamma_{I}\right\}=1-F_{\left|h_{\mathrm{SE}}\right|^{2}}$ $\left(\gamma_{I} / \psi\right)$. Using the CDF of $\left|h_{S E}\right|^{2}$ in (2), one obtains the 


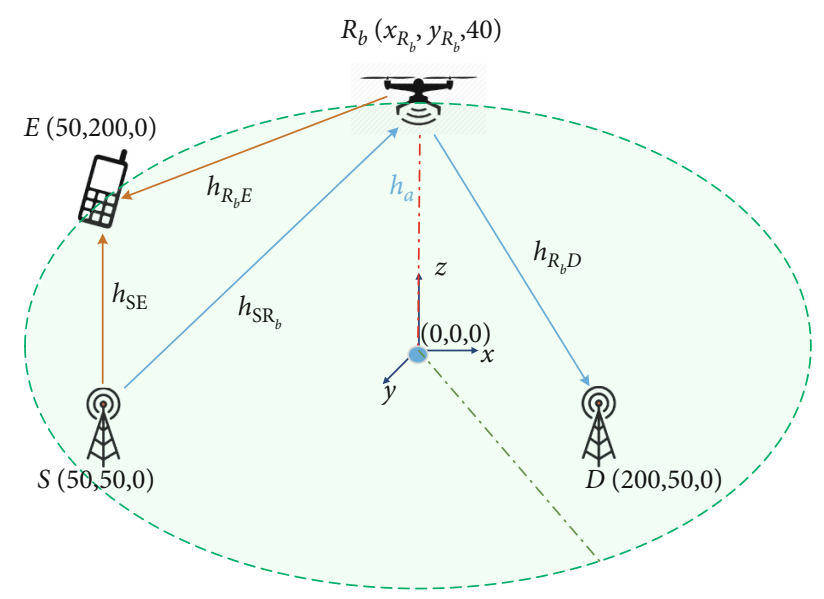

FIGURE 2: 3D model in UAV-aided relaying systems.

intercept probability of the energy harvesting UAV selection in the exact closed form (the expression in (18) is exact because it is derived from the exact $\gamma_{E o}$ in (10)) as

$$
\mathrm{IP}_{o}=1-\left(1-e^{-\gamma_{I} / \psi \bar{\gamma}_{\mathrm{SE}}}\right)=e^{-\gamma_{I} / \psi \bar{\gamma}_{\mathrm{SE}}}
$$

3.2. Scenario 2: E Receives Signals from $S$ in the Second Phase and $R_{b}$ in the Third Phase. In this scenario, the eavesdropper can combine both signals $\left(y_{\mathrm{SE}}\right.$ and $\left.y_{R_{b} E}\right)$ received from $S$ in the second phase and $R_{b}$ in the third phase by the SC technique or the MRC technique. The IP for the SC technique is given by $\mathrm{IP}_{\mathrm{sc}}=\operatorname{Pr}\left\{\gamma_{\mathrm{Esc}} \geq \gamma_{I}\right\}=1-\operatorname{Pr}\left\{\max \left(\gamma_{\mathrm{SE}}, \gamma_{R_{b} E}\right)<\gamma_{I}\right\}$ , while that for the MRC technique is $\operatorname{IP}_{m r c}=\operatorname{Pr}\left\{\gamma_{E m r c} \geq \gamma_{I}\right\}$ $=1-\operatorname{Pr}\left\{\gamma_{\mathrm{SE}}+\gamma_{R_{b} E}<\gamma_{I}\right\}$.

Lemma 3. The tight approximated closed-form representation (the expression in (19) is approximated because it is derived from the approximated $\gamma_{R_{b} E}$ in (13). Nonetheless, the approximation in (13) is tight, and, hence, (19) matches simulation results as shown in Section 5) of the intercept probability for the SC technique is given by

$$
\begin{aligned}
I P_{s c} \simeq 1-\left(1-e^{-\gamma_{I} / \bar{\gamma}_{S E} \psi}\right) & {\left[1-\frac{2 M}{\psi \bar{\gamma}_{S R}} \sum_{q=0}^{M-1}(-1)^{q} C_{M-1}^{q} e^{-(q+1) \gamma_{I} / \psi \bar{\gamma}_{S R}}\right.} \\
& \left.\sqrt{\frac{\gamma_{I} \psi \bar{\gamma}_{S R}}{\xi \eta \bar{\gamma}_{R E}(q+1)}} K_{1}\left(2 \sqrt{\frac{\gamma_{I}(q+1)}{\xi \eta \psi \bar{\gamma}_{R E} \bar{\gamma}_{S R}}}\right)\right] .
\end{aligned}
$$

Proof. Since all the links are independent, the IP for the SC technique can be rewritten as

$\mathrm{IP}_{\mathrm{sc}}=1-\operatorname{Pr}\left\{\gamma_{\mathrm{SE}}<\gamma_{I}\right\} \operatorname{Pr}\left\{\gamma_{R_{b} E}<\gamma_{I}\right\}=1-F_{\left|h_{\mathrm{SE}}\right|^{2}}\left(\frac{\gamma_{I}}{\psi}\right) F_{\gamma_{R_{b} E}}\left(\gamma_{I}\right)$.

The first term in (20), $F_{\left|h_{S E}\right|^{2}}\left(\gamma_{I} / \psi\right)$, is the CDF of $\left|h_{\mathrm{SE}}\right|^{2}$ evaluated at $\gamma_{I} / \psi$. Using (2), this term is straightforwardly

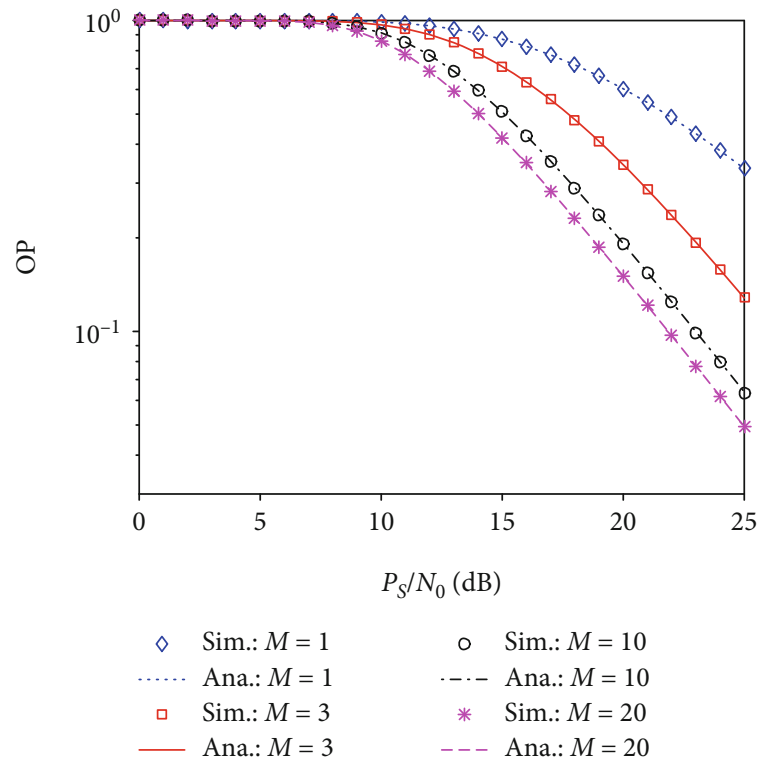

FIGURE 3: Outage probability versus the normalized SNR.

computed as $1-e^{-\gamma_{I} / \bar{\gamma}_{\mathrm{SE}} \psi}$. Meanwhile, the second term in (20), $F_{\gamma_{R_{b} E}}\left(\gamma_{I}\right)$, is the CDF of $\gamma_{R_{b} E}$ evaluated at $\gamma_{I}$. Because $\gamma_{R_{b} E}$ is the SNR of the $S-R_{\mathrm{b}}-E$ communication link, it has the same form as the SNR of the $S-R_{b}-D$ communication link, $\gamma_{D}$ in (9). Therefore, by substituting $D$ with $E$ in (17), one obtains the tight approximated closed-form expression of $F_{\gamma_{R_{b} E}}\left(\gamma_{I}\right)$. Plugging these results into (20), one reduces (20) to (19). This completes the proof.

Lemma 4. The tight approximated closed-form representation of the intercept probability for the MRC technique is given by

$$
\begin{aligned}
& I P_{m r c} \simeq e^{-\gamma_{I} / \psi \bar{\gamma}_{S E}}\left\{1+\frac{M}{\psi^{3 / 2} \bar{\gamma}_{S E} \sqrt{\xi \eta \bar{\gamma}_{S R} \bar{\gamma}_{R E}}} \sum_{q=0}^{M-1} \sum_{n \geq 0} \sum_{j=0}^{n}(-1)^{q} C_{M-1}^{q}\right. \\
& \left.\times \frac{2^{1+j}(-1)^{j} \sqrt{\pi} \Gamma(2) \Gamma(n-0.5) L(n, j)}{\Gamma(-0.5) \Gamma(1.5+n) n !(q+1)^{1-j / 2} B^{1-j}} \Theta\left(\sqrt{\gamma_{I}}, j+1, \frac{q+1}{\psi \bar{\gamma}_{S R}}-\frac{1}{\psi \bar{\gamma}_{S E}}, B \sqrt{q+1}\right)\right\},
\end{aligned}
$$

where $L(n, j)=\left(\begin{array}{c}n-1 \\ j-1\end{array}\right) n ! / j !$ with $n, j \in \aleph$ is Lah numbers, $\Gamma(\cdot)$ is the Gamma function, and $\gamma(\cdot, \cdot)$ is the lower incomplete Gamma function,

$\Theta(u, a, b, c)= \begin{cases}c^{-a-1} \gamma(a+1, a c) & , b=0, \\ e^{c^{2} / 4 b} \sum_{m=0}^{a} C_{a}^{m} \frac{(-c / 2 / b)^{a-m}}{b^{(m+1) / 2} 2}\left[\gamma\left(\frac{m+1}{2}, b\left\{u+\frac{c}{2 b}\right\}^{2}\right)-\gamma\left(\frac{m+1}{2}, \frac{c^{2}}{4 b}\right)\right] & , b \neq 0,\end{cases}$

$$
B=\frac{2}{\sqrt{\xi \eta \psi \bar{\gamma}_{R E} \bar{\gamma}_{S R}}}
$$

Proof. Please see Appendix C. 


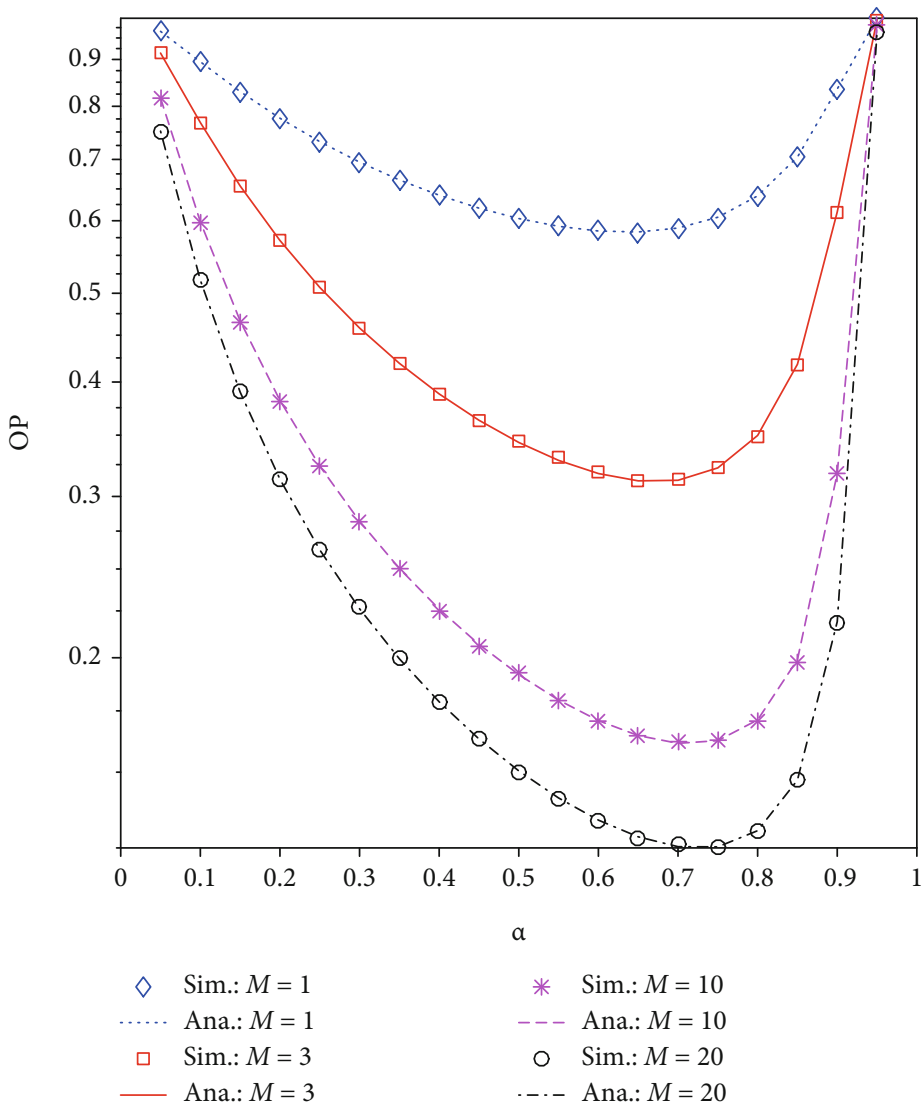

FIGURE 4: Outage probability versus the time splitting factor.

\section{Protected Zone of UAVs}

The "protected zone" is defined as a zone in which the secrecy of the legitimate transmitter is enhanced by decreasing the capacity of the wire-tapped channel while the reliability of the desired end-to-end connection is maintained at an acceptable level. Different from terrestrial communications where the locations of the relays may be fixed (relays may be base stations in mobile communications), in the context of UAVaided communications, in this paper, the UAVs which play roles as relays are moving. Therefore, an optimization problem should be formulated to find optimal locations to deploy UAVs for reliable and secured legitimate communications.

For ease of presentation, it is assumed that the source station $S$, the destination station $D$, and the eavesdropper $E$ are fixed on the 2D ground plane, while only UAVs are above the ground plane with the height of $h_{a}$ as shown in Figure 2. This means that the $3 \mathrm{D}$ coordinate of $v$ with $v=\{S, D, E\}$ can be denoted as $\left(x_{v}, y_{v}, 0\right)$, while that of the UAV $u$ is $\left(x_{u}\right.$, $\left.y_{u}, h_{a}\right)$. Then, the distance of the air-to-ground (or groundto-air) communication link $u-v$ is $d_{u v}=\sqrt{h_{a}^{2}+r_{u v}^{2}}$ in which $r_{u v}=\sqrt{\left(x_{u}-x_{v}\right)^{2}+\left(y_{u}-y_{v}\right)^{2}}$.

Now, the protected zone is specifically defined in the context of UAV-aided communications as the region of the best $\mathrm{UAV}, Z_{\omega_{R_{b}}}$, over which the outage probability of $D$ is less than a threshold $\mathrm{OP}_{\text {th }}$ to guarantee the reliability of the $S-$
$R_{b}-D$ communication link, while the intercept probability of $E$ is also smaller than a certain level $\mathrm{IP}_{\text {th }}$ to secure that link. Therefore, the optimization problem to find the protected zone can be formulated as

$$
Z_{\omega_{R_{b}}}=\left\{\left(x_{R_{b}}, y_{R_{b}}\right)\right\}
$$

subject to

$$
\begin{aligned}
& \mathrm{OP}_{\omega_{R_{b}}} \leq \mathrm{OP}_{\text {th }}, \\
& \mathrm{IP}_{\omega_{R_{b}}} \leq \mathrm{IP}_{\mathrm{th}},
\end{aligned}
$$

where $\mathrm{IP}_{w_{R_{b}}}$ and $\mathrm{OP}_{w_{R_{b}}}$ are, respectively, the intercept probability of $E$ and the outage probability of $D$ when the best UAV $R_{b}$ is in the protected zone. In other words, the protected zone is a set of the locations, $\left\{\left(x_{R_{b}}, y_{R_{b}}\right)\right\}$, of the best UAV $R_{b}$ such that (25) and (26) are satisfied.

Due to the complexity of the expressions for $\mathrm{IP}_{w_{R_{b}}}$ and $\mathrm{O}$ $\mathrm{P}_{w_{R_{b}}}$ as shown in the previous section, it is hard to reach the exact closed-form solution for the protected zone $Z_{\omega_{R_{b}}}$. However, thanks to the tight approximated and exact closed-form expressions of $\mathrm{IP}_{w_{R_{b}}}$ and $\mathrm{OP}_{w_{R_{b}}}$, an exhaustive search can be performed to numerically and quickly find $Z_{\omega_{R_{b}}}$ in the above 


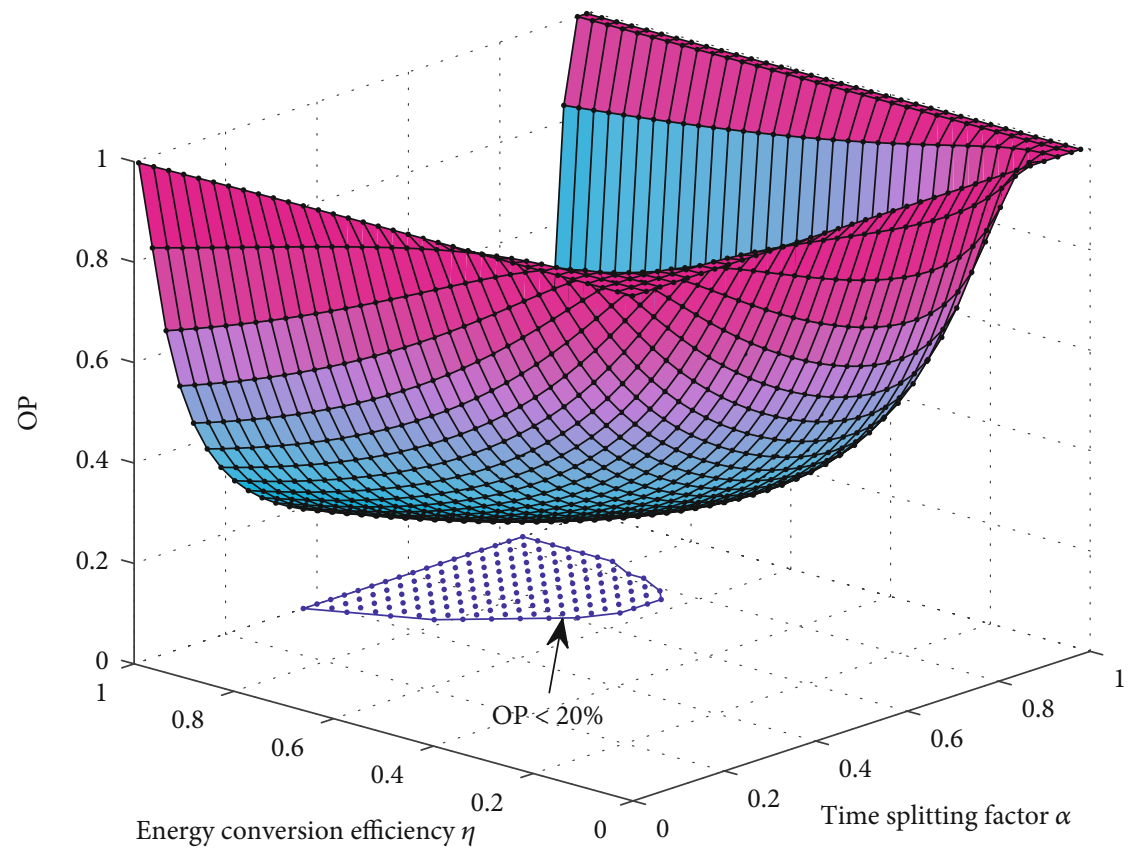

FIGURE 5: Outage probability versus $\alpha$ and $\eta$.

optimization problem. The next section will present some useful results from this exhaustive search.

\section{Illustrative Results}

In this section, Monte-Carlo simulations are conducted to obtain simulation results in order to prove the validity of the proposed theoretical expressions in (17), (18), (19), and (21) as well as to have insights into the secrecy and the reliability of the energy harvesting UAV selection. First, the end-to-end connection reliability is analyzed by investigating the outage probability. Then, the secrecy performance of the UAVaided relaying system is examined in terms of the intercept probability. Some simulation parameters fixed throughout this section are the carrier frequency $f_{C}=900 \mathrm{MHz}$ and the pathloss exponent $\tau=2$ (corresponding to free-space path loss).

Figure 3 illustrates the outage probability of the legitimate channel from $S$ through $R_{b}$ to $D$ with respect to the normalized SNR $\psi=P_{S} / N_{0}$ when the distances of the $\left(S-R_{i}, R_{i}-D\right)$ links are $d_{S R}=100 \mathrm{~m}$ and $d_{R D}=50 \mathrm{~m}$, respectively; the time splitting factor is $\alpha=0.5$; the energy conversion efficiency is $\eta=$ 0.8 ; the required spectral efficiency is $\mathscr{C}_{\text {th }}=0.1 \mathrm{bps} / \mathrm{Hz}$. This figure shows the coincidence between the analysis and the simulation, validating the proposed expression in (17). Additionally, the reliability of the $S-R_{b}-D$ communication link is improved with increasing the normalized SNR as expected. Moreover, the efficiency of the UAV selection is proven, which is based on the observation in Figure 3 that the more number of UAVs is, the lower outage probability is achievable.

Figure 4 represents the impact of the time for energy scavenging on the outage probability of $D$ for the same parameters as Figure 3 except $\psi=P_{S} / N_{0}=20 \mathrm{~dB}$. This figure validates the proposed expression in (17) due to the coincidence between the analysis and the simulation. Additionally, the OP decreases with increasing the time for energy harvesting (i.e.,

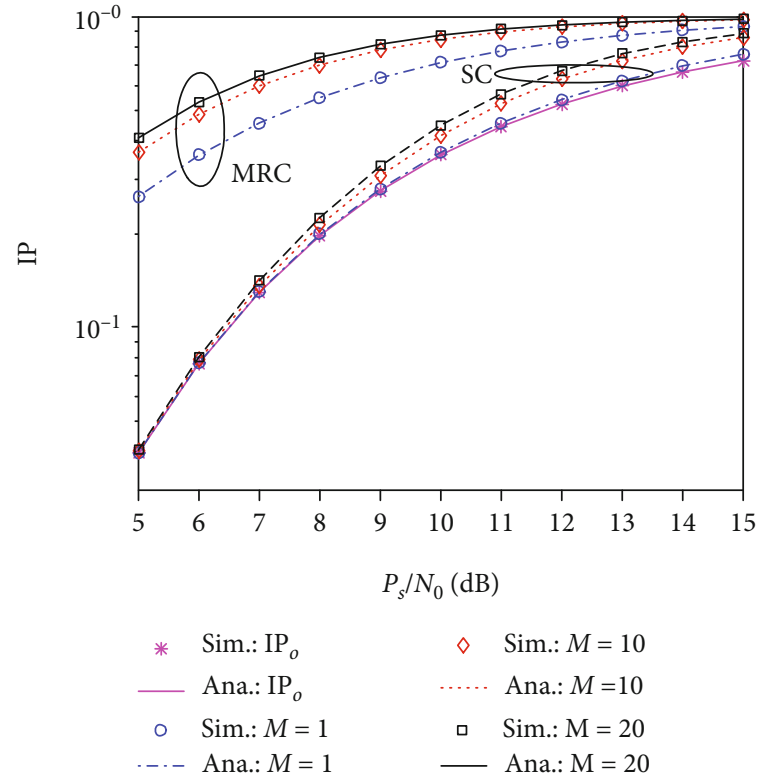

FIGURE 6: Intercept probability versus the normalized SNR.

increasing $\alpha$ ). This is because the UAVs harvest more energy to keep the reliable connection to $D$ when $\alpha$ increases. However, the more time for energy harvesting, the less time for message transmission, causing lower channel capacity in the third phase which eventually increases the OP. Therefore, when the time splitting factor is greater than a certain value (e.g., $\alpha=0.7$ for $M=10$ in Figure 4), the $\mathrm{OP}$ increases with increasing $\alpha$. As such, $\alpha$ should be optimized to achieve reliable communication. Furthermore, the transmission reliability is improved with deploying more UAVs, showing the efficiency of the UAV selection. 


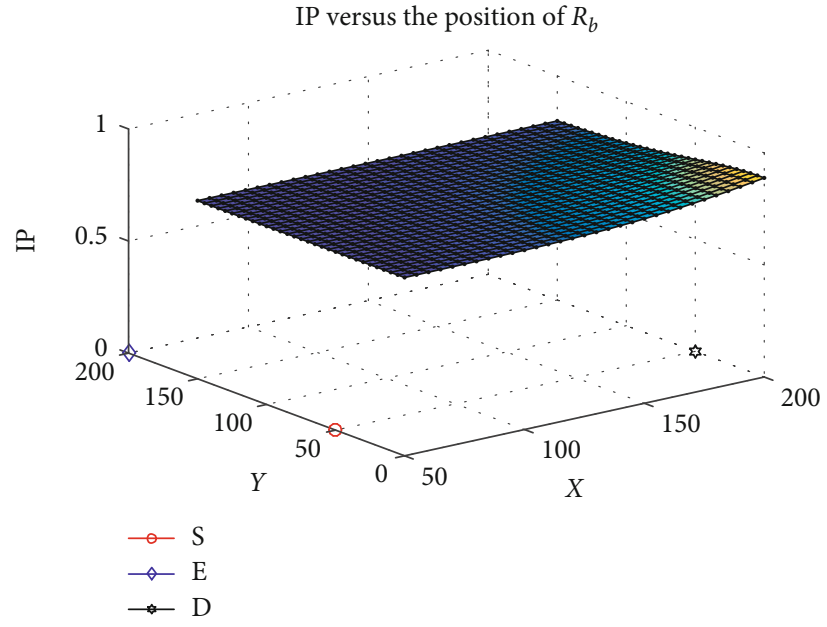

(a)

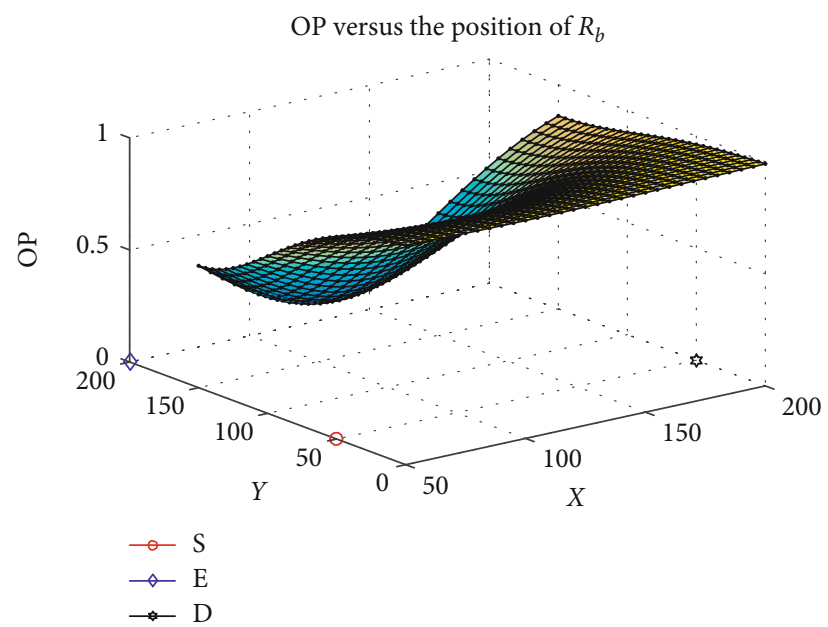

(c)

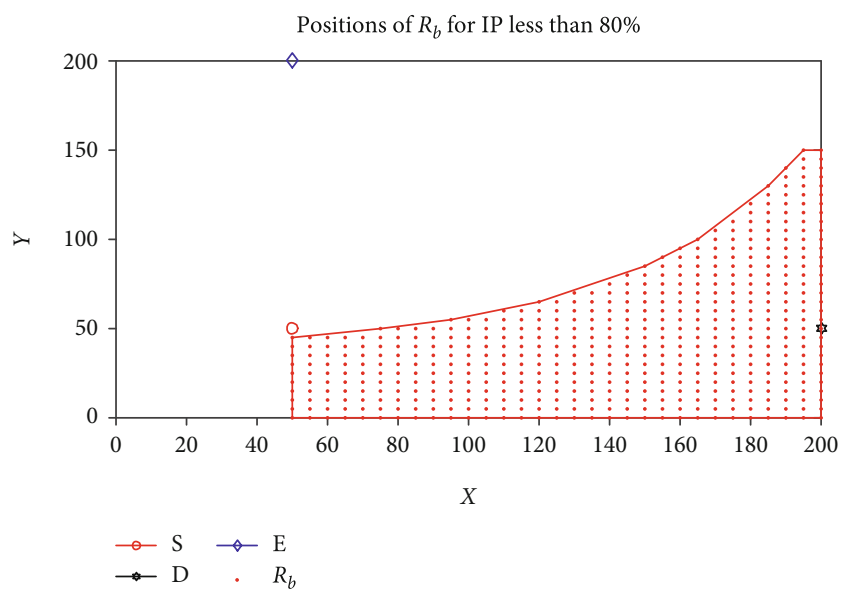

(b)

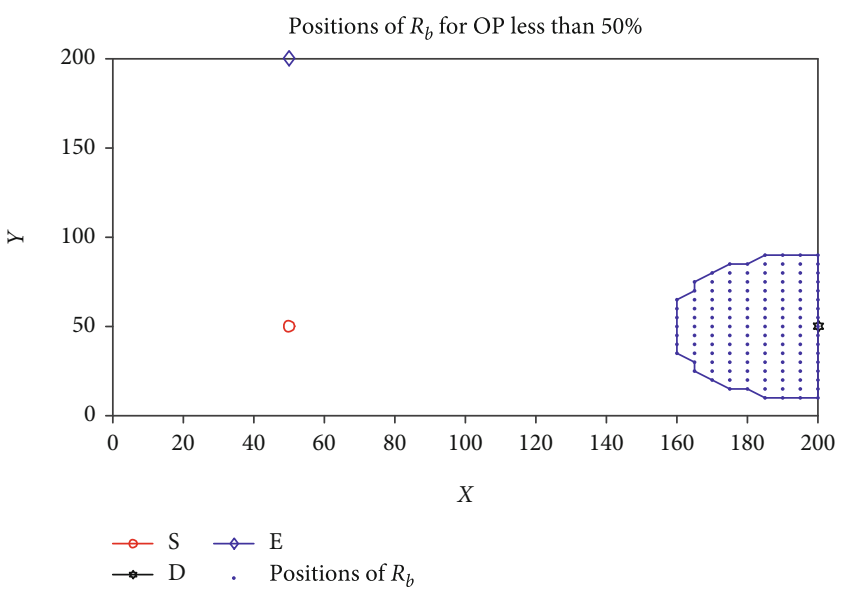

(d)

Figure 7: Illustration of finding the protected zone of UAVs.

Besides the time splitting factor, the energy conversion efficiency is another important factor, which affects the amount of the harvested energy, eventually impacting the system performance. Figure 5 demonstrates the OP versus both $\eta$ and $\alpha$ for the same parameters as Figure 4 except $M=10$. Results show that conditioned on $\alpha$, the higher $\eta$, the smaller the OP. This is reasonable because increasing $\eta$ helps UAVs harvest more energy, and, hence, UAVs transmit signals with higher power for lower OP. For ease of observing the effect of both $\eta$ and $\alpha$ on the OP, we find the region on the $(\eta, \alpha)$ plane in which the OP is less than a certain level for acceptable connection reliability. Figure 5 demonstrates this region in which the OP is lower than $20 \%$. It is seen that in order to achieve an acceptable connection reliability (e.g., OP $<20 \%$ ), the efficiency of the energy harvester must be greater than a certain value (e.g., $\eta$ $>0.63$ ) and the time fraction required for scavenging energy must fall in a certain range (e.g., $0.37<\alpha<0.87$ ). This result can be interpreted by combining the result of the OP versus $\alpha$ in Figure 4 and the fact that increasing $\eta$ reduces the OP.

To illustrate the secrecy performance of the UAV selection, the intercept probability is investigated for different availability degrees of eavesdropped information at the eavesdropper. Figure 6 demonstrates the IP with respect to the normalized SNR for the same parameters as Figure 3 except $\mathscr{C}_{I}=0.1$ $\mathrm{bps} / \mathrm{Hz}$ and the distances of the $\left(S-E, R_{i}-E\right)$ links of $d_{S E}=$ $100 \mathrm{~m}$ and $d_{R E}=50 \mathrm{~m}$, respectively. It is recalled that without the eavesdropped information in the third phase, the IP is IP in (18) independent of the number of UAVs $M$, while with that information in the third phase, the IP is $\mathrm{IP}_{s c}$ in (19) for the SC technique and IP ${ }_{m r c}$ in (21) for the MRC technique. Both IP and IP ${ }_{m r c}$ depend on the number of UAVs $M$. It is seen that the simulation coincides with the analysis, confirming the exactness of the proposed IP expressions. In addition, information transmission is less secured when the number of UAVs increases, showing the negative side of the UAV selection where only information of the $S-R_{i}$ communication link is employed to select the UAV as the relay without paying attention to $E$. Moreover, the availability degree of eavesdropped information obviously benefits $E$ in stealing legitimate information. More specifically, $\mathrm{IP}_{o}$ is always smaller than $\mathrm{IP}_{\mathrm{sc}}$ and $\mathrm{IP}_{m r c}$ , as expected. Furthermore, it is well-known that the MRC technique has better performance than the SC one. This explains 
the results in Figure 6 that $\mathrm{IP}_{m r c}$ is significantly greater than I $\mathrm{P}_{\mathrm{sc}}$. In other words, the eavesdropper is the most effective in intercepting the transmission of $S$ with the MRC technique. Additionally, the intercept performance of $E$ is improved with increasing the normalized SNR.

Figure 7 illustrates how to find the protected zone of UAVs where $\mathrm{OP}_{\text {th }}=0.5, \mathrm{IP}_{\text {th }}=0.8, P_{S} / N_{0}=20 \mathrm{~dB}, \alpha=0.5, \eta$ $=0.8, \mathscr{C}_{I}=\mathscr{C}_{t h}=0.1 \mathrm{bps} / \mathrm{Hz}$, and the coordinates of terminals as shown in Figure 2. Figure 7(a) shows the IP with respect to the positions of the best UAV $R_{b}$ (the projected coordinate of $R_{b}$ on the $2 \mathrm{D}$ ground plane is denoted by $(X, Y)$ in Figure 7$)$ when the SC technique is employed at $E$. From this figure and by the exhaustive search, the positions of $R_{b}$ such that the IP is less than 0.8 (i.e., (26) holds) can be found and shown in Figure 7(b). Similarly, Figure 7(c) shows the OP with respect to the positions of the best UAV $R_{b}$, and by the exhaustive search, the positions of $R_{b}$ such that the OP is less than 0.5 (i.e., (25) holds) can be found and shown in Figure 7(d). By intersecting both regions of $R_{b}$ in Figures 7(b) and 7(d), the protected zone can be found straightforwardly. In this illustration, the intersection (i.e., the protected zone) is also the region of $R_{b}$ in Figure 7(d). This protected zone is to guarantee the reliable connection to $D$ while preventing $E$ from eavesdropping. As such, the protected zone in Figure 7(d) is reasonable because it is close to $D$ for reliable signal reception but far from $E$ for higher security.

\section{Conclusions}

This paper investigated an UAV-aided relaying system under the eavesdropping of the wire-tapper where only one UAV among multiple UAVs, all with capability of harvesting energy from RF signals, is selected as a relay. The system performance was analyzed in terms of the outage probability of the destination station and the intercept probability of the eavesdropper through the proposed tight approximated and exact closed-form expressions. Different availability degrees of eavesdropped information at the eavesdropper were also considered to evaluate various information security levels that the system may encounter. The analysis was verified by the Monte-Carlo simulation. Multiple results showed the efficiency of the UAV selection in keeping reliable connection over long distance. By taking advantage of UAVs' high mobility, this paper also determined the protected zone of the selected UAV to maintain the security and the reliability at an acceptable level. Furthermore, the results demonstrated that the time splitting factor for the energy harvesting process can be optimized to achieve the best performance.

\section{Appendix}

\section{A. Proof of Lemma 1}

The PDF of $X$ is defined as

$$
F_{X}(x)=\operatorname{Pr}\{X<x\}=\operatorname{Pr}\left\{\max _{i \in \Phi_{R}}\left|h_{\mathrm{SR}_{i}}\right|^{2}<x\right\}
$$

Since all the $S-R_{i}$ communication links are independent,
(A.1) is rewritten as

$$
F_{X}(x)=\prod_{i=1}^{M} \operatorname{Pr}\left\{\left|h_{\mathrm{SR}_{i}}\right|^{2}<x\right\}=\left[F_{\left.\left|h_{\mathrm{SR}_{i}}\right|^{2}(x)\right]^{M}} .\right.
$$

Inserting the CDF of $\left|h_{\mathrm{SR}_{i}}\right|^{2}$ given in (2) into (A.2) with a notice that $\bar{\gamma}_{S R_{i}}=\bar{\gamma}_{S R}$ due to the homogeneous distribution of UAVs, one obtains

$$
F_{X}(x)=\left(1-e^{-x / \bar{\gamma}_{\mathrm{SR}}}\right)^{M}
$$

Using the binomial expansion $(a+b)^{n}=\sum_{m=0}^{n} C_{n}^{m} a^{m} b^{n-m}$ , one reduces (A.3) to (14), completing one part of the proof.

Taking the derivative of (14) with respect to $x$, one obtains the PDF of $X$ which is exactly the same as (15), completing the rest of the proof.

\section{B. Proof of Lemma 2}

The detailed derivation of the tight approximated closedform expression of the outage probability in (17) is as follows. First, we rewrite (16) as

$$
F_{\gamma_{D}}\left(\gamma_{\mathrm{th}}\right) \simeq \operatorname{Pr}\left\{\xi \eta Y\left(X-\frac{\gamma_{\mathrm{th}}}{\psi}\right)<\frac{\gamma_{\mathrm{th}}}{\psi}\right\} .
$$

Equation (B.1) can be simplified by considering two cases subject to $X$ as

$$
F_{\gamma_{D}}\left(\gamma_{\text {th }} \mid X\right) \simeq \begin{cases}\operatorname{Pr}\left\{Y<\frac{\gamma_{\text {th }}}{\xi \eta\left(\psi X-\gamma_{\text {th }}\right)}\right\}, & X \geq \frac{\gamma_{\text {th }}}{\psi}, \\ 1, & X<\frac{\gamma_{\text {th }}}{\psi} .\end{cases}
$$

As such, the unconditional outage probability is expressed as

$F_{\gamma_{D}}\left(\gamma_{\mathrm{th}}\right) \simeq \int_{0}^{\gamma_{\mathrm{th}} / \psi} f_{X}(x) d x+\int_{\gamma_{\mathrm{th}} / \psi}^{\infty} F_{Y}\left(\frac{\gamma_{\mathrm{th}}}{\xi \eta\left(\psi x-\gamma_{\mathrm{th}}\right)}\right) f_{X}(x) d x$.

Plugging $F_{Y}(x)$ in (2) into the above equation and after some simplifications, one can further simplify (B.3) as

$$
\begin{aligned}
& F_{\gamma_{D}}\left(\gamma_{\mathrm{th}}\right) \simeq \int_{0}^{\gamma_{\mathrm{th}} / \psi} f_{X}(x) d x+\int_{\gamma_{\mathrm{th}} / \psi}^{\infty}\left(1-e^{-\gamma_{\mathrm{th}} / \xi \bar{\gamma}_{\mathrm{RD}}\left(\psi x-\gamma_{\mathrm{th}}\right)}\right) f_{X}(x) d x \\
& =1-\int_{\gamma_{\mathrm{th}} / \psi}^{\infty} e^{-\gamma_{\mathrm{th}} / \xi \eta \bar{\gamma}_{\mathrm{RD}}\left(\psi x-\gamma_{\mathrm{th}}\right)} \mathrm{f}_{X}(x) d x .
\end{aligned}
$$

It is noted that $\bar{\gamma}_{R_{i} D}=\bar{\gamma}_{\mathrm{RD}}, i \in \Phi_{R}$, due to the homogeneous distribution of UAVs. Therefore, $F_{Y}(x)=1-e^{-x / \bar{\gamma}_{\mathrm{RD}}}$ is used in (B.4). 
Now, plugging $f_{X}(x)$ in (15) into (B.4), one obtains

$F_{\gamma_{D}}\left(\gamma_{\mathrm{th}}\right) \simeq 1-\frac{M}{\bar{\gamma}_{S R}} \sum_{q=0}^{M-1}(-1)^{q} C_{M-1}^{q} \int_{\gamma_{\mathrm{th}} / \psi}^{\infty} e^{-\left(\gamma_{\mathrm{th}} / \xi \bar{\xi} \bar{\gamma}_{\mathrm{RD}}\left(\psi x-\gamma_{\mathrm{th}}\right)+(q+1) / \bar{\gamma}_{\mathrm{SR}} x\right)} d x$.

By changing variable $t=x \psi-\gamma_{\text {th }}$, (B.5) becomes

$\left.F_{\gamma_{D}}\left(\gamma_{\mathrm{th}}\right) \simeq 1-\frac{M}{\psi} \bar{\gamma}_{\mathrm{SR}} \sum_{q=0}^{M-1}(-1)^{q} C_{M-1}^{q} e^{-(q+1) \gamma_{\mathrm{t}} / \bar{\gamma}_{\mathrm{SR}} \psi} \int_{0}^{\infty} e^{-\left(\gamma_{\mathrm{th}} / \xi \bar{\xi} \bar{\gamma}_{\mathrm{RD}} t+(q+1) t / \psi \bar{\gamma}_{\mathrm{SR}}\right.}\right) d t$.

Using $\int_{0}^{\infty} e^{-\beta / 4 x-\gamma x} d x=\sqrt{\beta / \gamma} K_{1}(\sqrt{\beta \gamma})$ in ([19], eq. (3.324.1)) to compute the last integral in (B.6), one can represent (B.6) in the tight approximated closed form as (17). This completes the proof.

\section{Proof of Lemma 3}

The IP for the MRC technique can be rewritten as

$$
\mathrm{IP}_{m r c}=1-\operatorname{Pr}\left\{\gamma_{R_{b} E}<\gamma_{I}-\gamma_{\mathrm{SE}}\right\}=1-\int_{0}^{\gamma_{I}} F_{\gamma_{R_{b} E}}\left(\gamma_{I}-x\right) f_{\gamma_{\mathrm{SE}}}(x) d x .
$$

Using (1), one obtains $f_{\gamma_{\mathrm{SE}}}(x)$ in (C.1). Meanwhile, by following the proof of Lemma 3, one also obtains $F_{\gamma_{R_{b} E}}(\cdot)$ by changing $D$ to $E$ in (17). Inserting all the results into (C.1) and after some simplifications, we have

$$
\begin{aligned}
& \mathrm{IP}_{m r c} \simeq 1-\int_{0}^{\gamma_{I}} \frac{1}{\psi \bar{\gamma}_{S E}} e^{-x / \psi \bar{\gamma}_{\mathrm{SE}}} d x+\frac{2 M}{\psi^{2} \bar{\gamma}_{S E} \bar{\gamma}_{S R}} \sum_{q=0}^{M-1}(-1)^{q} C_{M-1}^{\mathrm{q}} e^{-(q+1) \gamma_{I} / \psi \bar{\gamma}_{S R}} \\
& \underbrace{\underbrace{\frac{\psi \bar{\gamma}_{S R}\left(\gamma_{I}-x\right)}{(q+1) \xi \eta \bar{\gamma}_{R E}}}}_{\int_{0}^{\gamma_{1}} e^{\left.((q+1)) \bar{\gamma}_{S R}-1 / \bar{\gamma}_{\mathrm{SE}}\right) x / \psi}} K_{1}\left(2 \sqrt{\frac{(q+1)\left(\gamma_{I}-x\right)}{\xi \eta \psi \bar{\gamma}_{S R} \bar{\gamma}_{R E}}}\right) d x
\end{aligned}
$$
form as

The term $J_{1}$ in (C.2) is easily expressed in the exact closed

$$
J_{1}=1-e^{-\gamma_{1} / \psi \bar{\gamma}_{\mathrm{SE}}} .
$$

By changing the variable $y=\gamma_{I}-x$ in the term $J_{2}$ in (C.2), one obtains

$$
J_{2}=e^{\left.((q+1)) \bar{\gamma}_{\mathrm{SR}}-1 / \bar{\gamma}_{\mathrm{SE}}\right) \gamma_{I} / \psi} \int_{0}^{\gamma_{I}} e^{-\left((q+1) / \bar{\gamma}_{\mathrm{SR}}-1 / \bar{\gamma}_{\mathrm{SE}}\right) y / \psi} \sqrt{\frac{\psi \bar{\gamma}_{S R} y}{\xi \eta(q+1) \bar{\gamma}_{R E}}} K_{1}\left(2 \sqrt{\frac{(q+1) y}{\xi \eta \psi \bar{\gamma}_{R E} \bar{\gamma}_{S R}}}\right) d y .
$$

Using series representation for the modified Bessel function of the second kind in ([20], eq. (1.3)) to express $K_{1}(x)$ as

$$
K_{1}(x)=\frac{e^{-x}}{x} \sum_{n \geq 0} \sum_{j=0}^{n} \frac{(-1)^{j} \sqrt{\pi} \Gamma(2) \Gamma(n-0.5) L(n, j)}{2^{1-j} \Gamma(-0.5) \Gamma(1.5+n) n !} x^{j},
$$

one can rewrite (C.4) in the series form as

$$
\begin{aligned}
J_{2}= & e^{\left((q+1) / \bar{\gamma}_{\mathrm{SR}}-1 / \bar{\gamma}_{\mathrm{SE}}\right) \gamma_{1} / \psi} \sqrt{\frac{\psi \bar{\gamma}_{S R}}{\xi} \bar{\zeta}_{R E n \geq 0}} \sum_{j=0}^{n} \frac{(-1)^{j} \sqrt{\pi} \Gamma(2) \Gamma(n-0.5) L(n, j) B^{j-1}}{2^{1-j} \Gamma(-0.5) \Gamma(1.5+n) n !(q+1)^{1-j / 2}} \\
& \times \int_{0}^{\gamma_{1}} y^{j / 2} e^{-\left(((q+1)) \bar{\gamma}_{\mathrm{SR}}-1 / \bar{\gamma}_{\mathrm{SE}}\right) y / \psi-B \sqrt{(q+1))}} d y,
\end{aligned}
$$

where $B$ is given in (23).

By changing the variable $t=\sqrt{y}$, one can rewrite the term $J_{3}$ in (C.6) as

$$
\begin{aligned}
J_{3}= & 2 \int_{0}^{\sqrt{\gamma_{I}}} t^{j+1} e^{-\left((q+1) / \bar{\gamma}_{\mathrm{SR}}-1 / \bar{\gamma}_{\mathrm{SE}}\right) t^{2} / \psi-B \sqrt{q+1} t} d t \\
& =2 \Theta\left(\sqrt{\gamma_{I}}, j+1, \frac{q+1}{\psi \bar{\gamma}_{\mathrm{SR}}}-\frac{1}{\psi \bar{\gamma}_{\mathrm{SE}}}, B \sqrt{q+1}\right),
\end{aligned}
$$

where

$$
\Theta(u, a, b, c)=\int_{0}^{u} x^{a} e^{-b x^{2}-c x} d x .
$$

Inserting (C.7) into (C.6) and then plugging (C.6) and (C.3) into (C.2), the intercept probability in (C.2) for the MRC technique matches (21). Therefore, the last step to complete the proof is to prove that (C.8) can be expressed in the exact closed form as (22). This step proceeds as follows. First, (C.8) is rewritten in a compact form as

$$
\Theta(u, a, b, c)= \begin{cases}U_{1}, & b=0, \\ U_{2}, & b \neq 0\end{cases}
$$

where

$$
\begin{gathered}
U_{1}=\int_{0}^{u} x^{a} e^{-c x} d x, \\
U_{2}=e^{c^{2} / 4 b} \int_{0}^{u} x^{a} e^{-b(x+c / 2 b)^{2}} d x .
\end{gathered}
$$
form as

Using ([19], eq. (3.351.1)), $U_{1}$ is expressed in exact closed

$$
U_{1}=c^{-a-1} \gamma(a+1, a c) .
$$

Then changing the variable $y=x+c / 2 b$, one rewrites $U_{2}$ as

$$
U_{2}=e^{c^{2} / 4 b} \int_{c / 2 b}^{u+c / 2 b}\left(y-\frac{c}{2 b}\right)^{a} e^{-b y^{2}} d y
$$


Applying the binomial expansion to $(y-c / 2 b)^{a},(\mathrm{C} .12)$ is further simplified as

$$
\begin{aligned}
U_{2}= & e^{c^{2} / 4 b} \int_{c / 2 b}^{u+c / 2 b}\left(\sum_{m=0}^{a} C_{a}^{m} y^{m}\left(-\frac{c}{2 b}\right)^{a-m}\right) e^{-b y^{2}} d y \\
& =e^{c^{2} / 4 b} \sum_{m=0}^{a} C_{a}^{m}\left(-\frac{c}{2 b}\right)^{a-m} \underbrace{\int_{\frac{c}{2 b}}^{u+c / 2 b} y^{m} e^{-b y^{2}} d y}_{I}
\end{aligned}
$$

Changing the variable $y=\sqrt{x}$, one rewrites the last integral in (C.13) as

$$
\begin{aligned}
I= & \frac{1}{2} \int_{(c / 2 b)^{2}}^{(u+c / 2 b)^{2}} x^{(m+1) / 2-1} e^{-b x} d x \\
& =\frac{1}{2}\left[\int_{0}^{(u+c / 2 b)^{2}} x^{(m+1) / 2-1} e^{-b x} d x-\int_{0}^{(c / 2 b)^{2}} x^{(m+1) / 2-1} e^{-b x} d x\right]
\end{aligned}
$$

Applying $\int_{0}^{u} \bowtie x^{\nu-1} e^{-\mu x} d x=\mu^{-v} \gamma(v, \mu u)$ in ([19], eq. (3.381.1)), each integral in (C.14) can be expressed in the exact closed form. This leads to

$I=\frac{b^{-(m+1) / 2}}{2}\left[\gamma\left(\frac{m+1}{2}, b\left\{u+\frac{c}{2 b}\right\}^{2}\right)-\gamma\left(\frac{m+1}{2}, b\left\{\frac{c}{2 b}\right\}^{2}\right)\right]$.

Plugging (C.15) into (C.13), one obtains the exact closed form of $U_{2}$ as

$U_{2}=e^{c^{2} / 4 b} \sum_{m=0}^{a} C_{a}^{m} \frac{(-c / 2 / b)^{a-m}}{b^{(m+1) / 2} 2}\left[\gamma\left(\frac{m+1}{2}, b\left\{u+\frac{c}{2 b}\right\}^{2}\right)-\gamma\left(\frac{m+1}{2}, \frac{c^{2}}{4 b}\right)\right]$

Inserting (C.11) and (C.16) into (C.9), $\Theta(u, a, b, c)$ in (C.9) is exactly the same as (22). This completes the proof.

\section{Outlines of Derivations for Heterogeneous Locations of UAVs}

This appendix outlines how to derive the IP and OP expressions for the case that UAVs are located heterogeneously.

The key derivation to obtain the tight approximated closed-form expression of the outage probability in (17) is to solve the integral in (B.4). Apparently, this integral is solved in the exact closed form if the PDF of $X$ is expressed as a linear combination of the exponential functions of the variable $x$ as in (15). Therefore, what we need to obtain the tight approximated closed-form expression of the outage probability for the case that UAVs are located heterogeneously is to prove that the PDF of $X$ is expressed as a linear combination of the exponential functions of the variable $x$.
Towards this end, we rewrite (A.2) as

$$
F_{X}(x)=\prod_{i=1}^{M}\left(1-e^{-x / \bar{\gamma}_{\mathrm{SR}_{i}}}\right)
$$

It is recalled from $([21]$, eq. (86)) that

$$
\prod_{k \in \Phi_{R}}\left(1-a_{k}\right)=1+(-1)^{\left|\Phi_{R}\right|} \prod_{k \in \Phi_{R}} a_{k}+\sum_{v=1}^{\left|\Phi_{R}\right|-1}(-1)^{v} \sum_{s_{1}=1}^{\left|\Phi_{R}\right|-v+1} \sum_{s_{2}=s_{1}+1}^{\left|\Phi_{R}\right|-v+2} \cdots \sum_{s_{v}=s_{v-1}+1}^{\left|\Phi_{R}\right|} \prod_{k \in A} a_{k},
$$

where $\left|\Phi_{R}\right|$ is the cardinality of $\Phi_{R}$ and $A=\left\{\Phi_{R}\left[s_{1}\right], \Phi_{R}\left[s_{2}\right]\right.$ $\left., \cdots, \Phi_{R}\left[s_{v}\right]\right\}$ with $\Phi_{R}[i]$ denoting the $i^{\text {th }}$ element in the set $\Phi_{R}$.

Using (D.2) to expand (D.1) as

$$
\begin{aligned}
& F_{X}(x)=1+(-1)^{\left|\Phi_{R}\right|} \prod_{k \in \Phi_{R}} e^{-x /\rangle_{S R_{k}}}+\sum_{v=1}^{\left|\Phi_{R}\right|-1}(-1)^{v} \sum_{s_{1}=1}^{\left|\Phi_{R}\right|-v+1} \sum_{s_{2}=S_{1}+1}^{\left|\Phi_{R}\right|-v+2} \cdots \sum_{s_{v}=s_{v-1}+1}^{\left|\Phi_{R}\right|} \prod_{k \in A} e^{-x / \gamma_{S_{S R_{k}}}}
\end{aligned}
$$

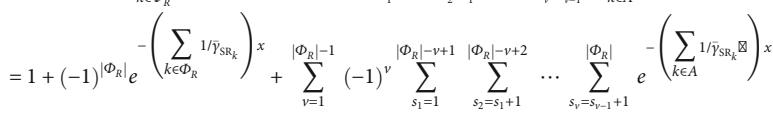

Taking the derivative of (D.3) with respect to $x$, one obtains the PDF of $X$ as

$$
\begin{aligned}
f_{X}(x)= & (-1)^{\left|\Phi_{R}\right|+1}\left(\sum_{k \in \Phi_{R}} \frac{1}{\bar{\gamma}_{S R_{k}}}\right) e^{-\left(\sum_{k \in \Phi_{R}} 1 / \bar{\gamma}_{S R_{k}}\right) x} \\
& +\sum_{v=1}^{\left|\Phi_{R}\right|-1}(-1)^{v+1} \sum_{s_{1}=1}^{\left|\Phi_{R}\right|-v+1} \sum_{s_{2}=s_{1}+1}^{\left|\Phi_{R}\right|-v+2} \cdots \sum_{s_{v}=s_{v-1}+1}^{\left|\Phi_{R}\right|}\left(\sum_{k \in A} \frac{1}{\bar{\gamma}_{S R_{k}}}\right) e^{-\left(\sum_{k \in A} 1 / \bar{\gamma}_{\mathrm{SR}_{k}}\right) x} .
\end{aligned}
$$

It is obvious that the PDF of $X$ in (D.4) is a linear combination of the exponential functions of the variable $x$. Therefore, the tight approximated closed-form expression of the outage probability for the case that UAVs are located heterogeneously can be derived straightforwardly.

Similarly, the key derivation to obtain the tight approximated closed-form expressions of the intercept probability in (19) and (21) is to achieve the CDF of $\gamma_{R_{b} E}, F_{\gamma_{R_{b} E}}(x)$, in order to derive (20) and (C.1). Because $\gamma_{R_{b} E}$ in (13) has the same form as $\gamma_{D}$ in (9) and the CDF of $\gamma_{D}, F_{\gamma_{D}}(x)=\mathrm{OP}$, is obtained for the case that UAVs are located heterogeneously, $F_{{R_{b} E}}(x)$ is derived from $F_{\gamma_{D}}(x)$ by substituting $D$ with $E$. Therefore, given $F_{\gamma_{R_{b}}}(x)$ for the case that UAVs are located heterogeneously, one obtains straightforwardly the tight approximated closed-form expressions of $\mathrm{IP}_{\mathrm{sc}}$ and $\mathrm{IP}_{m r c}$ for this case.

\section{Data Availability}

The authors declare that all data used to support the findings of this study are included within the article 


\section{Conflicts of Interest}

The authors declare that they have no conflicts of interest.

\section{Acknowledgments}

This research is funded by Vietnam National University HoChiMinh City (VNU-HCM) under grant number B2021-20-01. We would like to thank Ho Chi Minh City University of Technology (HCMUT), VNU-HCM for the support of time and facilities for this study.

\section{References}

[1] N. P. Santos, V. Lobo, and A. Bernardino, "Directional statistics for 3d model-based uav tracking," IEEE Access, vol. 8, pp. 33884-33897, 2020.

[2] M. Mozaffari, W. Saad, M. Bennis, Y. Nam, and M. Debbah, “A tutorial on UAVs for wireless networks: applications, challenges, and open problems," IEEE Communications Surveys \& Tutorials, vol. 21, no. 3, pp. 2334-2360, 2019.

[3] L. Ji, J. Chen, and Z. Feng, "Spectrum allocation and performance analysis for backhauling of UAV assisted cellular network," China Communications, vol. 16, no. 8, pp. 83-92, 2019.

[4] M. J. Sobouti, Z. Rahimi, A. H. Mohajerzadeh et al., "Efficient deployment of small cell base stations mounted on unmanned aerial vehicles for the internet of things infrastructure," IEEE Sensors Journal, vol. 20, no. 13, pp. 7460-7471, 2020.

[5] M. Hayashi and A. Vazquez-Castro, "Two-way physical layer security protocol for Gaussian channels," IEEE Transactions on Communications, vol. 68, no. 5, pp. 3068-3078, 2020.

[6] A. M. Abdelhady, O. Amin, B. Shihada, and M. Alouini, "Spectral efficiency and energy harvesting in multi-cell slipt systems," IEEE Transactions on Wireless Communications, vol. 19, no. 5, pp. 3304-3318, 2020.

[7] H. Sun, M. Naraghi-Pour, W. Sheng, and R. Zhang, "A hopby-hop relay selection strategy in multi-hop cognitive relay networks," IEEE Access, vol. 8, pp. 21117-21126, 2020.

[8] A. Omri and M. O. Hasna, "Physical layer security analysis of UAV based communication networks," in 2018 IEEE 88th vehicular technology conference (VTC-fall), pp. 1-6, Chicago, IL, USA, 2018.

[9] G. Zhang, Q. Wu, M. Cui, and R. Zhang, "Securing UAV communications via joint trajectory and power control," IEEE Transactions on Wireless Communications, vol. 18, no. 2, pp. 1376-1389, 2019.

[10] D. Wang, B. Bai, G. Zhang, and Z. Han, "Optimal placement of low-altitude aerial base station for securing communications," IEEE Wireless Communications Letters, vol. 8, no. 3, pp. 869872, 2019.

[11] J. Ye, C. Zhang, H. Lei, G. Pan, and Z. Ding, "Secure UAV-toUAV systems with spatially random UAVs," IEEE Wireless Communications Letters, vol. 8, no. 2, pp. 564-567, 2019.

[12] Z. Sheng, H. D. Tuan, A. A. Nasir, T. Q. Duong, and H. V. Poor, "Secure UAV-enabled communication using HanKobayashi signaling," IEEE Transactions on Wireless Communications, vol. 19, no. 5, pp. 2905-2919, 2020.

[13] X. Yuan, Z. Feng, W. Ni, R. P. Liu, J. A. Zhang, and W. Xu, "Secrecy performance of terrestrial radio links under collaborative aerial eavesdropping," IEEE Transactions on Information Forensics and Security, vol. 15, pp. 604-619, 2020.
[14] R. Ma, W. Yang, Y. Zhang, J. Liu, and H. Shi, "Secure mmwave communication using UAV-enabled relay and cooperative jammer," IEEE Access, vol. 7, pp. 119729-119741, 2019.

[15] I. Y. Abualhaol and M. M. Matalgah, "Outage probability analysis in a cooperative UAVs network over Nakagami-m fading channels," in IEEE Vehicular Technology Conference, pp. 1-4, Montreal, QC, Canada, 2006.

[16] Y. Chen, N. Zhao, Z. Ding, and M. Alouini, "Multiple UAVs as relays: multi-hop single link versus multiple dual-hop links," IEEE Transactions on Wireless Communications, vol. 17, no. 9, pp. 6348-6359, 2018.

[17] S. Hanna, E. Krijestorac, H. Yan, and D. Cabric, "UAV swarms as amplify-and-forward MIMO relays," in 2019 IEEE 20th international workshop on signal processing advances in wireless communications (SPAWC), pp. 1-5, Cannes, France, 2019.

[18] B. Vucetic and J. Yuan, Space-Time Coding, John Wiley \& Sons, 2003.

[19] A. Jeffrey, D. Zwillinger, I. S. Gradshteyn, and I. M. Ryzhik, "34 - Definite integrals of elementary functions," in Table of Integrals, Series, and Products, D. Zwillinger, V. Moll, I. Gradshteyn, and I. Ryzhik, Eds., pp. 249-519, Academic Press, Boston, Eighth edition, 2015.

[20] D. Jankov Mairevi and T. K. Pogny, "On series representations for modified Bessel function of second kind of integer order," Integral Transforms and Special Functions, vol. 30, no. 3, pp. 181-189, 2019.

[21] K. Ho-Van, "Exact outage probability analysis of proactive relay selection in cognitive radio networks with MRC receivers," Journal of Communications and Networks, vol. 18, no. 3, pp. 288-298, 2016. 